\title{
Cryptocurrency Forecasting using $\alpha$-Sutte Indicator, ARIMA, and Long Short-Term Memory
}

\author{
Apriliyanus Rakhmadi Pratama ${ }^{1}$, Sigit Nugroho $^{2}$, Ketut Sukiyono ${ }^{3}$ \\ pratama.gokilz@gmail.com ${ }^{1}$, snugroho@unib.ac.id ${ }^{2}$,ksukiyono@unib.ac.id ${ }^{3}$ \\ Department of Statistics, University of Bengkulu, Bengkulu 38371, Indonesia ${ }^{1}$, Department of \\ Statistics, University of Bengkulu, Bengkulu 38371, Indonesia ${ }^{2}$, Department of Agricultural Economics, \\ University of Bengkulu, Bengkulu 38371, Indonesia ${ }^{3}$
}

\begin{abstract}
The purpose of these studies are to obtain bitcoin price predictions using three different approach in forecasting methods : ARIMA model, $\alpha$-sutte indicator and LSTM algorithm, and to find out the accuracy level of the three methods in forecasting bitcoin's price as well. Bitcoin closing's price each day taken from website of coin market starting from April 292013 to February 062019 was analyzed using R and Python softwares. Based on the smallest value of MSE, MAPE, and MAD, the LSTM algorithm gave the best prediction, followed by the $\alpha$-Sutte indicator and the ARIMA model.
\end{abstract}

Keywords: bitcoin, forecasting, a-Sutte indicator, ARIMA, LSTM.

\section{Introduction}

The instability of cryptocurrency bitcoin's price in the last few years encourages investor to find the most effective and efficient ways to make right the decision to achieve maximum profits. The uncertainty of bitcoin's price reflects that cryptocurrency has different system from the electronic money (e-money) that comes from the conventional banks. Unlike centralized conventional banks, cryptocurrency use decentralized control, which means the prices are not regulated by a single system rather than works through distributed ledger technology, typically a block chain, which serves as a public financial transaction database. It causes bitcoin's price being very volatile, dynamic and probabilistic. One way that can be used to overcome the problems is the forecasting approach. The forecasting approach theoretically offers many methods including Time Series Analysis, Neural Network, and $\alpha$ Sutte Indicator.

ARIMA models are one of the model in Time Series Analysis, stands for AutoRegressive Integrated Moving Average, with (p) as an Auto Regressive (AR) operator, the Integrated word refers to degrees of differencing (d) and (q) as a Moving Average (MA) operator [1]. ARIMA models are fitted to time series data either to better understand the data or to predict future points in the time series (forecasting). They are applied in some cases where data show evidence of non-stationery, where an initial differencing step can be applied one or more times to eliminate the non-stationery. Long Short Term-Memory algorithm is one of Neural Network algorithm, developed from Recurrent Neural Network (RNN) algorithm and popularized by Hochreiter and Schmidhuber [2]. The development is to overcome RNN algorithm problems that cause inaccurate result when analyzing long term data [3]. The $\alpha$-sutte indicator is the development of the sutte indicator by Ahmar [4] in 2017. Sutte indicator was 
originally developed based on the modification of the MA indicator by considering prices at the opening, closing, lowest and highest [5]. The $\alpha$-sutte indicator aims to increase the accuracy of forecasting and can be used to predict time series data.

\section{Notation}

\subsection{ARIMA Model}

The following is the general form of ARIMA models:

$$
\phi_{p}(B)(1-B)^{d} Z_{t}=\theta_{q}(B) \alpha_{t}
$$

where $Z_{t}$ is actual bitcoin's price at time $t, \phi_{p}$ is the $p$-th degree of AR operator, $\theta_{q}$ is the $q$-th degree of MA operator, $a_{t}$ is the error at time $t$, while $B$ is the backshift operator.

\subsection{LSTM Algorithm}

The LSTM algorithm is composed of an input layer, one or more hidden layers, and an output layer. The structure of LSTM memory cell is illustrated in Figure 1.Each memory cell has three types of gates, the forget gate, the input gate, and the output gate to maintain and adjust its cell state $s_{c}^{t}$. The forget gate $b_{\phi}^{t}$ defines which information is deleted from memory (cell state), the input gate $b_{\imath}^{t}$ specifies which information is added to the memory (cell state), the output gate $b_{\omega}^{t}$ specifies which information from memory (cell state) to be used as output information [5]. At the $t$-th step, LSTM decide what information will remove from the cell state through the decision by a transformation function (sigmoid and tanh) in forget layer. Every step to the forget layer, LSTM will decide what information will be removed or stored from the cell state. The forget layer will produce output in the form of numbers between 0 and 1 , where number 1 is interpreted as “completely keep this', while the number 0 is interpreted as "completely forget this" [5][6].

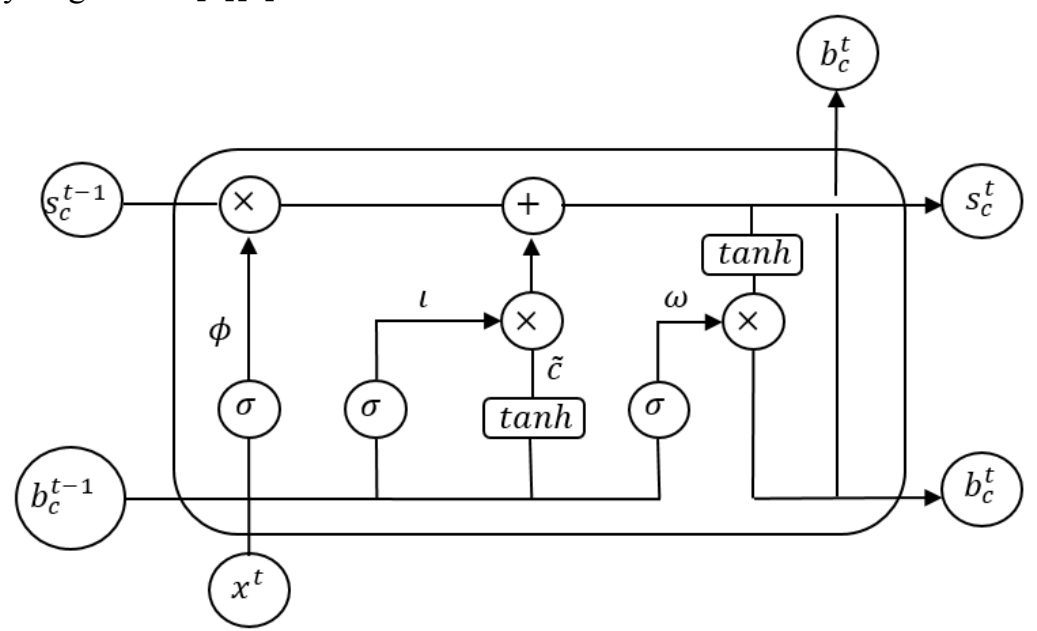

Fig.1. Memory Cell of LSTM algorithm 


\subsection{Alpha-Sutte Indicator}

The $\alpha$-sutte indicator is the development of the sutte indicator by Ahmar in 2017 which aims to increase the accuracy of forecasting and can be used to predict time series data. The $\alpha-$ sutte indicator has equation as followed:

$$
\alpha_{t}=(\alpha(2 \Delta x /(\alpha+\delta))+\beta(2 \Delta y /(\beta+\alpha))+\gamma(2 \Delta z /(\gamma+\beta))) / 3
$$

where $a_{t}=$ observation at time $\mathrm{t}, \delta=a_{t-4}, \alpha=a_{t-3}, \beta=a_{t-2}, \gamma=a_{t-1}, \Delta \mathrm{x}=\alpha-\delta, \Delta \mathrm{y}=\beta-\alpha$, and $\Delta \mathrm{z}=\gamma-\beta$.

\subsection{Performance evaluation indicators}

Mean Square Error (MSE), Mean Percentage Error (MAPE), and Mean Absolute Deviation are used to evaluate the forecasting performances.

$$
\begin{gathered}
M S E=\left(\sum_{t=1}^{n} Z_{t}\right) / n \\
M A P E=\sum_{t=1}^{n}\left|\left(Z_{t}-\hat{Z}_{t}\right) / Z_{t}\right| 100 \% / n \\
M A D=\sum_{t=1}^{n}\left|Z_{t}-\hat{Z}_{t}\right| / n
\end{gathered}
$$

where $Z_{t}$ is the actual bitcoin's price and $\hat{Z}_{t}$ is predicted price of bitcoin.

\section{Methods}

The data are the bitcoin closing's price each day starting from the period 29 April 2013 to 06 February 2019 obtained from the site www.coinmarketcap.com. They are divided into training data to form a model and testing data to test the accuracy of predictions from the model built. The number of training and testing data are 2104 and 7, respectively. From Figure 2 we can see that in the first 4 years bitcoin's price were pretty stable and low, then in the early 2017 bitcoin's price started to increase up until late 2017 reached its highest price at $\$ 19,497.4$. But then they continued to fall sharply.

\subsection{ARIMA Model Procedures}

a) Plot ACF and PACF on training data. The result shows in Figure 3. From the ACF plot, it shows a dies-down; while the PACF plot shows cut-off at lag-2. Therefore, the training data indicates an AR(2) model.

b) Stationary test was held on training data, the result of ADF's test indicate that the training data are not stationary.

c) Figure 4 shows that training data are not stationary in variances and mean. To overcome instability in variance, log transformation was held on the training data.

d) Then, transformed data will be differencing 1 time.

e) Redo stationary test on differenced data. From the ADF's test, it is said that the differenced data are stationary, as seen in Figure 5.

f) The plot of ACF and PACF stationary data are shown in Figure 6. As we can see, the plot show no significant lag period, so we cannot evaluate the number of periods of 
$p$ and $q$. Then, Python's syntax is used to find more candidate models with value of $p, d, q$ within range $0-4$.

g) Then, do white-noise test and residual test on best candidate model by smallest AIC's value.

h) Forecast using best ARIMA model.

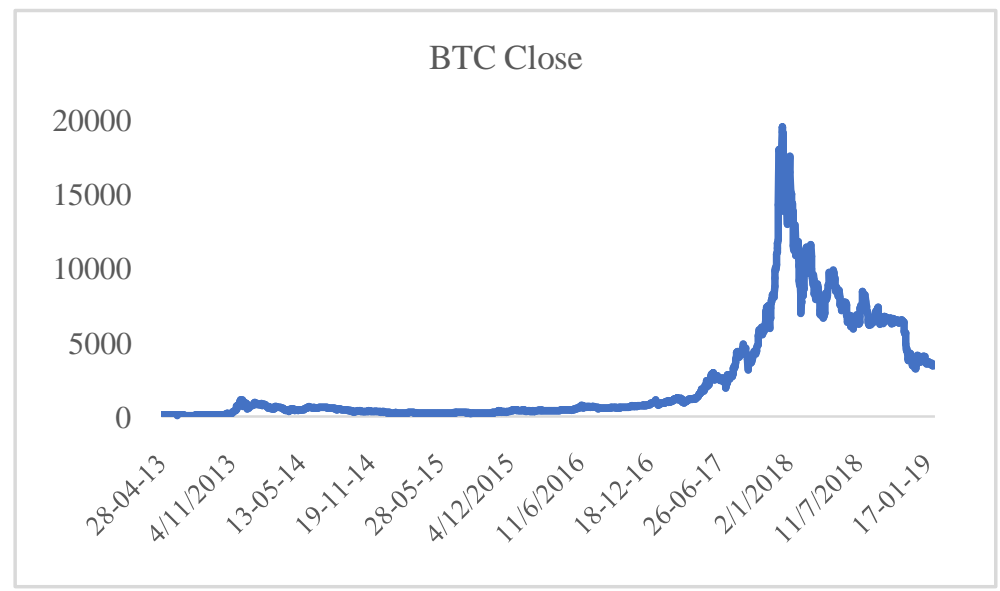

Fig.2. Plot of bitcoin's price each day from period April 292013 to February 062019
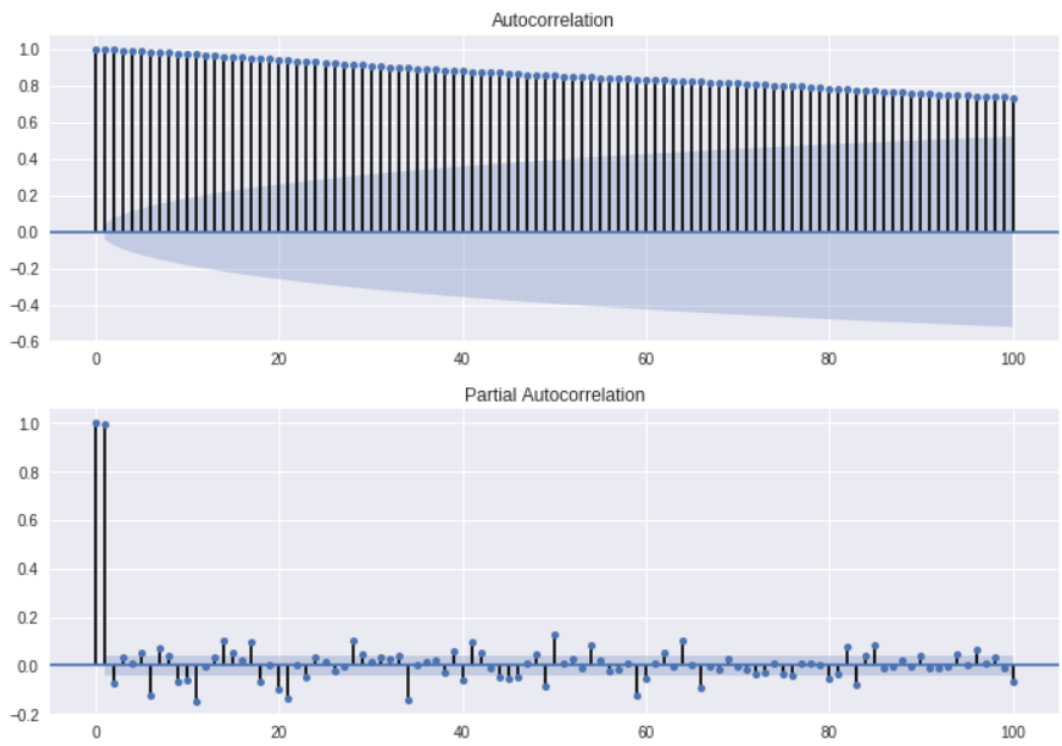

Fig.3. Plot ACF and PACF on training data 


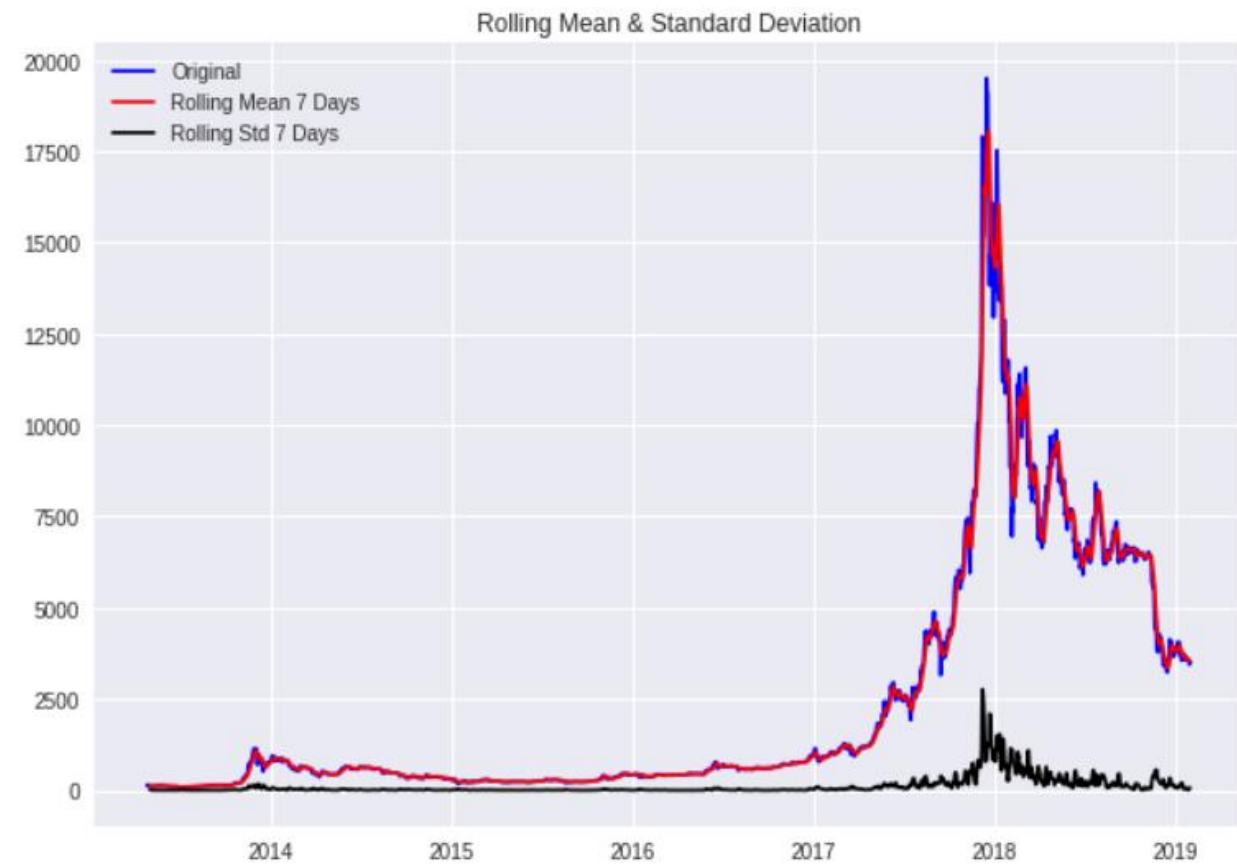

Fig.4. Plot Rolling Mean and Rolling Variance in 7 days of training data

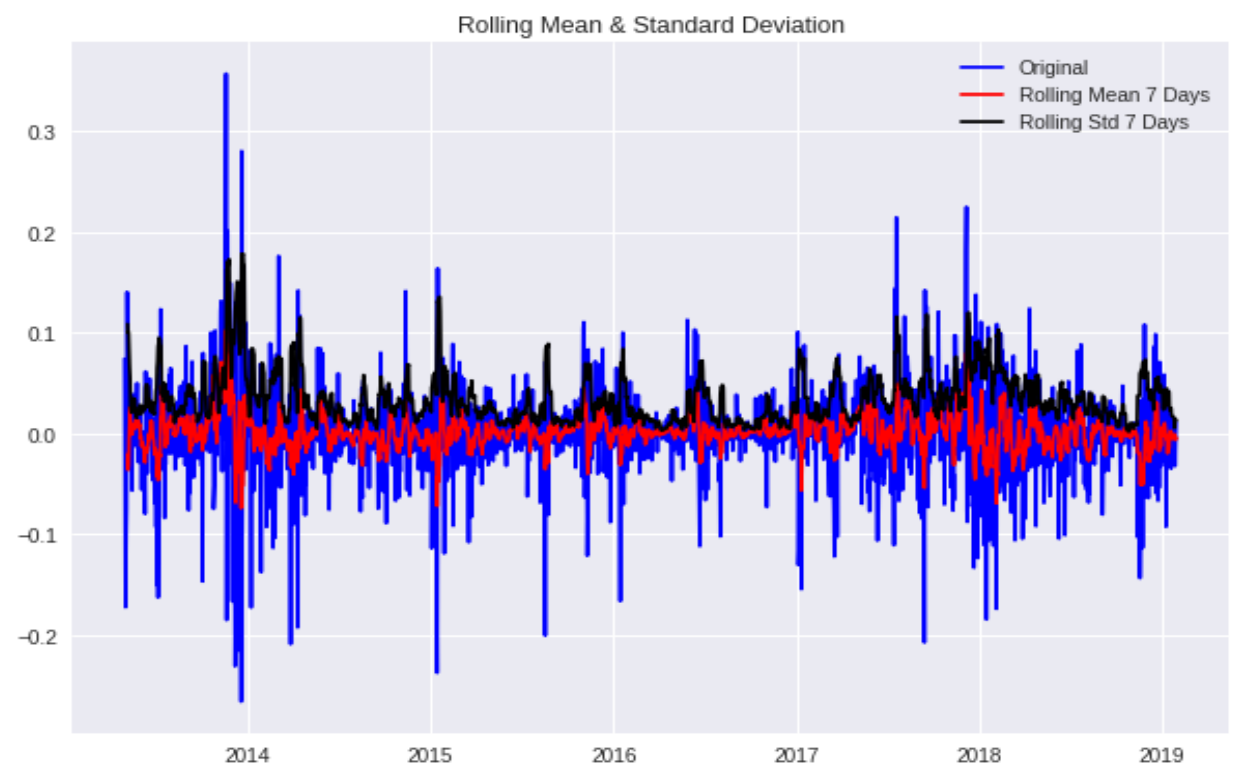

Fig.5. Plot Rolling Means and Rolling Variances in 7 days of differenced data 

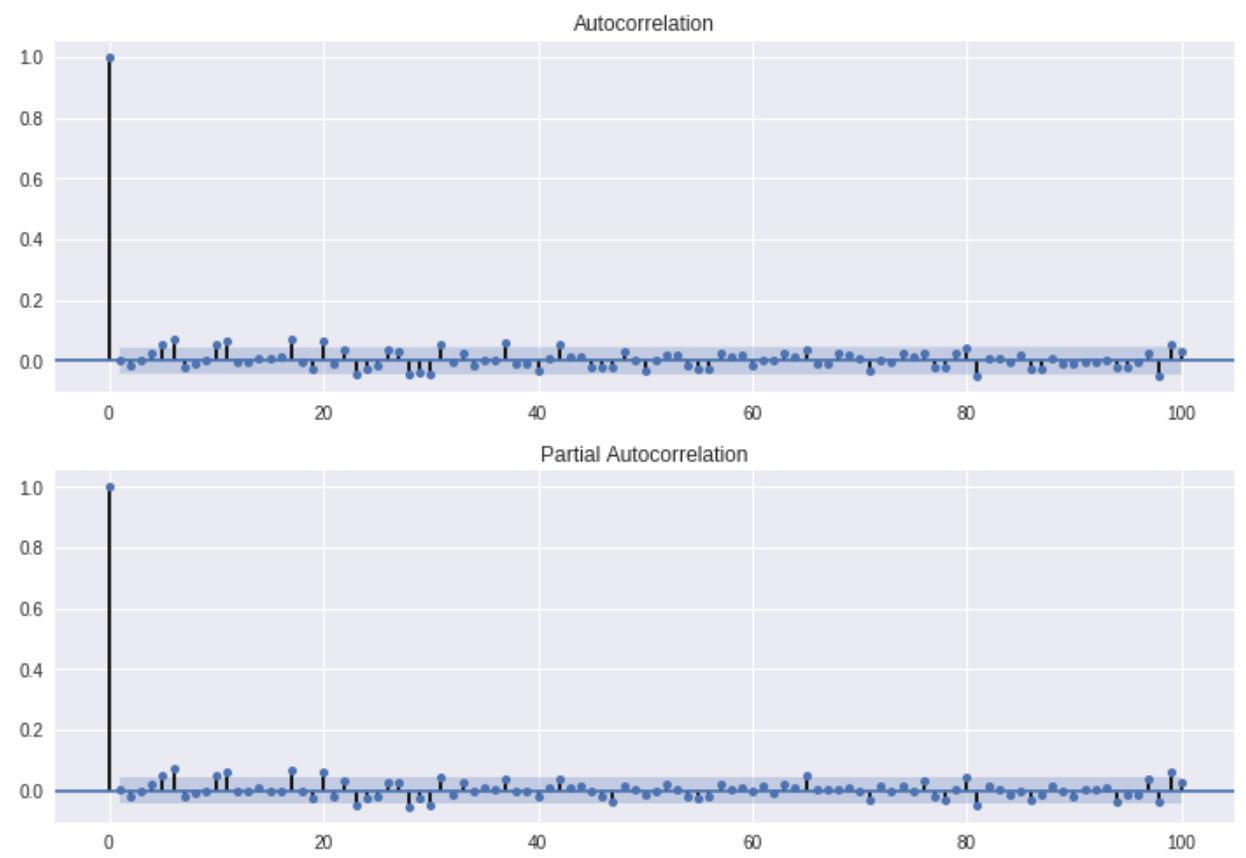

Fig.6. Plot ACF and PACF of differenced data

\subsection{LSTM Algorithm Procedure}

a) Training data will be normalize within range $[0,1]$ for easier computation.

b) Train the model using normalized training data, training process was held with help Keras Syntax.

c) Forecast using the obtained model.

d) Denormalize the forecasting results.

\section{3 $\alpha$-Sutte Indicator Procedure}

a) Calling library sutteForecastR.

b) Inputting training data on $\mathrm{R}$ program

c) Running syntax's program from library sutteForecastR on inputted data.

\section{Result and Discussion}

The accuracy of forecasting results from the three methods can be seen by comparing MSE, MAPE, and MAD criterion of each method. The higher the accuracy of forecasting, the lower the value of each criterion. These comparisons are shown in Table 1. 
Table 1. Accuracy level comparison of each criterion

\begin{tabular}{lrcc}
\hline \multicolumn{1}{r}{ Method } & MSE & MAPE & MAD \\
\hline ARIMA $(2,1,2)$ & 2532.718 & $1.266 \%$ & 43.761 \\
LSTM & $* \mathbf{4 2 1 . 6 8 6}$ & $* \mathbf{0 . 5 9 2} \%$ & $* \mathbf{2 0 . 5 3 5}$ \\
$\alpha$-Sutte Indicator & 1118.008 & $0.761 \%$ & 26.364 \\
\hline
\end{tabular}

Table 1 show that LSTM algorithm has the best accuracy level in forecasting bitcoin's price, followed by $\alpha$-Sutte Indicators and ARIMA models $(2,1,2)$.The forecasting results of each methods and its plot are shown in Table 2 and Figure 7.

Table 2. Forecasting result of three methods with full length data

\begin{tabular}{ccccc}
\hline \multirow{2}{*}{ Date } & Price & \multicolumn{3}{c}{ Predicted Price } \\
\cline { 2 - 5 } & & (ARIMA) & (LSTM) & (a-Sutte indicator) \\
\hline $2019-01-31$ & 3457.79 & 3485.713027 & 3437.243408 & 3454.286 \\
$2019-02-01$ & 3487.95 & 3478.178058 & 3467.436768 & 3453.702 \\
$2019-02-02$ & 3521.06 & 3479.668616 & 3500.587402 & 3501.379 \\
$2019-02-03$ & 3464.01 & 3494.809816 & 3443.469727 & 3532.821 \\
$2019-02-04$ & 3459.15 & 3511.329616 & 3438.604492 & 3466.334 \\
$2019-02-05$ & 3466.36 & 3515.425258 & 3445.822021 & 3449.759 \\
$2019-02-06$ & 3413.77 & 3508.963757 & 3393.181396 & 3448.286 \\
\hline
\end{tabular}

\section{Predicted Price}

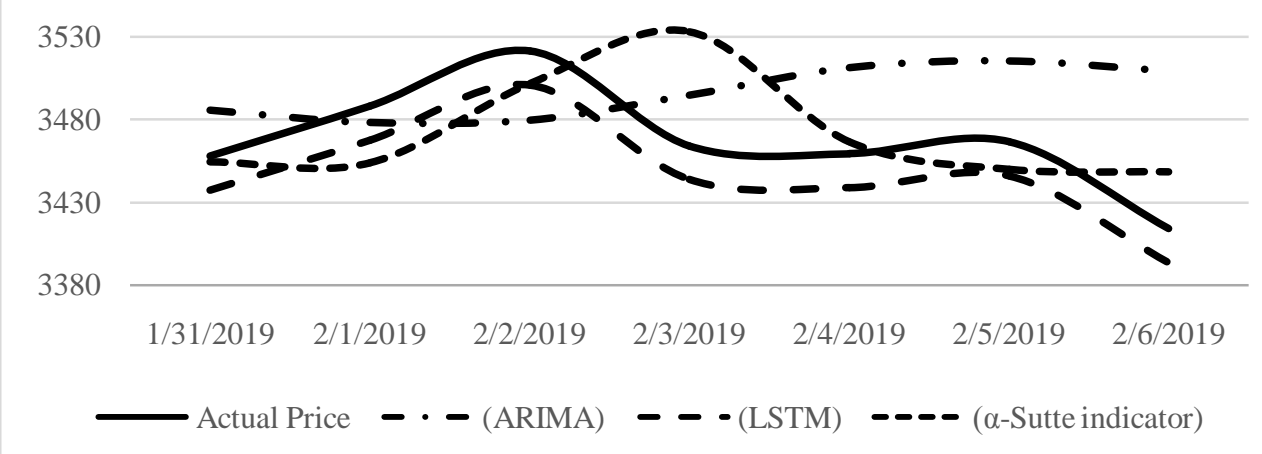

Fig.7. Plot of forecasting results from each method 
As we can see from Figure 6, LSTM predicted prices are able to follow the real price movement with a slight variance, while for $\alpha$-Sutte indicator, they are also trying to follow real price movement but with slightly off track on the $4^{\text {th }}$ day, and finally for the ARIMA model, they are failed to follow real price movement completely.

As seen in Figure 1, bitcoin's price has high volatility level. In order to overcome the volatility problem, training data will be cut and only used data starting from July 12017 until January 30 2019. The same forecasting process was carried out. Plot of new training data length is shown in Figure 8.

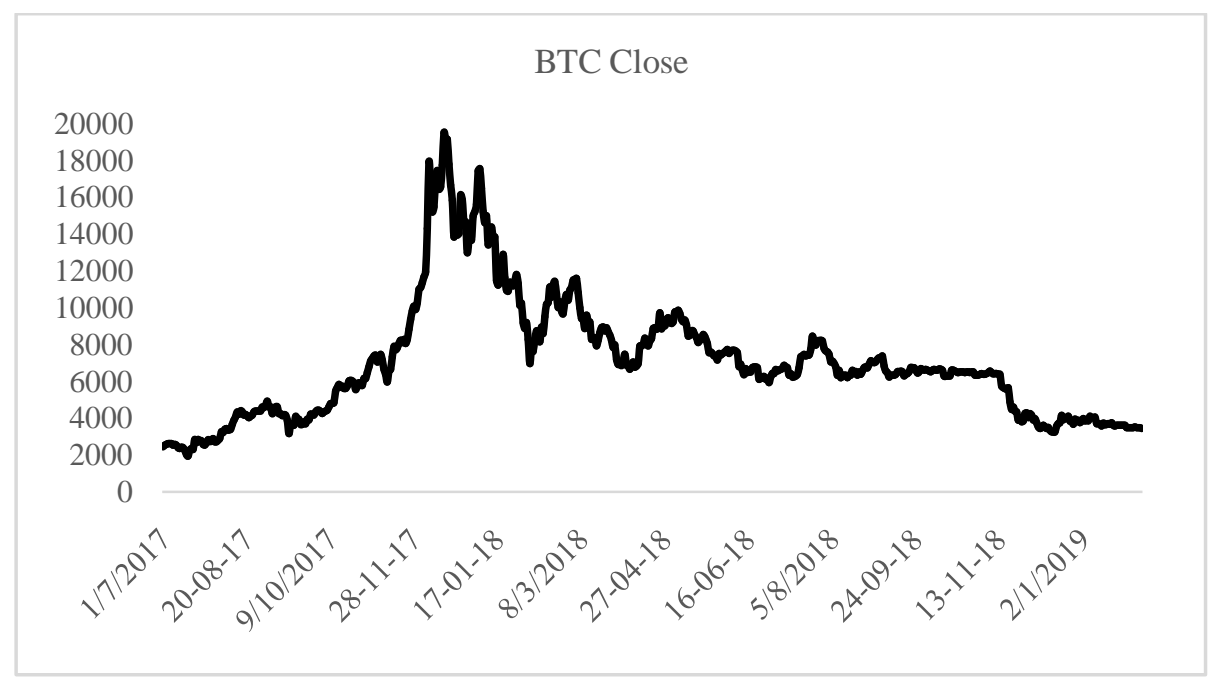

Fig.8. Plot of new length of Training Data in Table 3.

The accuracy of forecasting results from the three methods with new data can be seen

Table 3. Accuracy level comparison of each criterion with new length of Bitcoin's price data

\begin{tabular}{lrrr}
\multicolumn{1}{c}{ Method } & \multicolumn{1}{c}{ MSE } & \multicolumn{1}{c}{ MAPE } & \multicolumn{1}{l}{ MAD } \\
\hline ARIMA $(3,1,2)$ & 1755.761 & $1.006 \%$ & 34.705 \\
LSTM & $* \mathbf{5 . 5 4 9}$ & $* \mathbf{0 . 0 6 0} \%$ & $* \mathbf{2 . 0 7 7}$ \\
Indicator $\alpha$-Sutte & 1118.008 & $0.761 \%$ & 26.364 \\
\hline
\end{tabular}

Table 3 still shows that LSTM algorithm has the best accuracy level in forecasting bitcoin's price, also followed by $\alpha$-Sutte Indicators and ARIMA model $(3,1,2)$.

The forecasting results of each methods and its plot are shown in Table 4 and Figure 9. 
Table 4. Forecasting result of three methods with new length of Bitcoin's price data

\begin{tabular}{ccccc}
\hline \multirow{2}{*}{ Date } & \multirow{3}{*}{ Price } & \multicolumn{3}{c}{ Predicted Price } \\
\cline { 3 - 5 } & & $($ ARIMA) & $($ LSTM $)$ & (Indicator $\boldsymbol{\alpha}-$ Sutte $)$ \\
\hline $2019-01-31$ & 3457.79 & 3482.602722 & 3456.049561 & 3454.286 \\
$2019-02-01$ & 3487.95 & 3491.978792 & 3485.100342 & 3453.702 \\
$2019-02-02$ & 3521.06 & 3494.214721 & 3517.014893 & 3501.379 \\
$2019-02-03$ & 3464.01 & 3492.224930 & 3462.039062 & 3532.821 \\
$2019-02-04$ & 3459.15 & 3498.696435 & 3457.359131 & 3466.334 \\
$2019-02-05$ & 3466.36 & 3499.281838 & 3464.302490 & 3449.759 \\
$2019-02-06$ & 3413.77 & 3500.337364 & 3413.683350 & 3448.286 \\
\hline
\end{tabular}

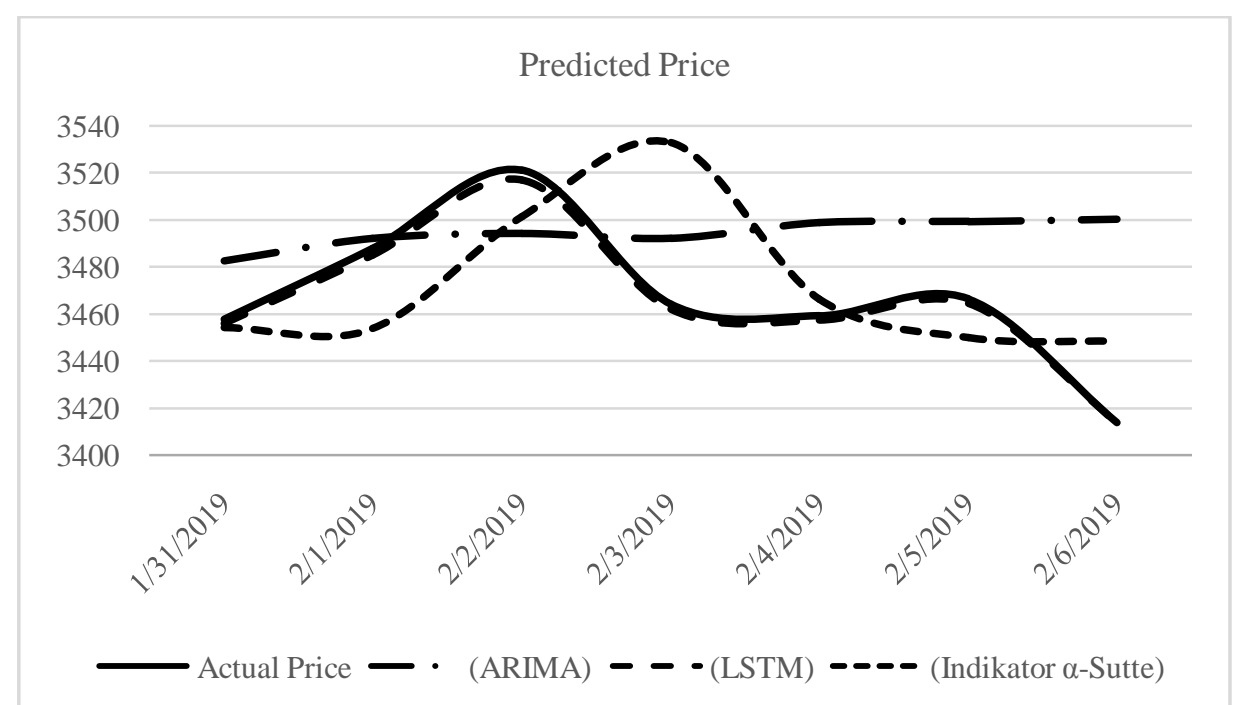

Figure 9. Plot of forecasting results from each method with new length of training data

As we can see at Figure 8, LSTM predicted price also able to follow real price's movement with much better variance than the last result, while $\alpha$-Sutte indicator still has same forecast result as before; and for the ARIMA model, it is still failed to follow real price movement, but having slightly better accuracy than the last result.

\section{Conclusion}

It is undeniable that the LSTM algorithm has a higher accuracy in predicting bitcoin's price compared to $\alpha$-sutte indicator and ARIMA model. Either using full length data or after cutting length of data, LSTM algorithm still has higher accuracy than the other methods by 
considering the MSE, MAPE, and MAD. This result indicates that the LSTM algorithm is more suitable for forecasting bitcoin's price compare other two methods. LSTM algorithm and ARIMA are sensitive to the length of data, while $\alpha$-Sutte Indicator is not.

\section{References}

[1] Box, G.E.P and Jenkins, G.M., Time Series Analysis: Forecasting and Control. Third Edition. Prentice Hall: New Jersey. (1994)

[2] Hochreiter, S., and Schmidhuber, J., Long Short-Term Memory, Neural Computation. (1997)

[3] Gers, F.A., Schmidhuber, J., and Cummins, F., Learning to Forget: Continual Prediction for LSTM, Neural Computation, 12(10),2451-2471, MIT Press, Doi: 10.1162/089976600300015015. (2000)

[4] Ahmar, A. S., Rahman, A., and Mulbar, U., $\alpha$-Sutte: A New Method for Timer Series Forecasting, J.Phys. Conf. Ser. 1040, 1 p.012018. (2017)

[5] T. Fischer and Krauss C., "Deep learning with long short-term memory networks for financial market predictions," European Journal of Operational Research, vol. 270, no. 2, pp. 654-669. (2018)

[6] Olah, C., "Understanding LSTM Networks," http://colah.github.io/posts/2015-08Understanding-LSTMs/, 2015. 